\title{
Micro/Nanofiber Optical Sensors
}

\author{
Lei ZHANG ${ }^{1}$, Jingyi $\mathrm{LOU}^{2}$, and Limin TONG ${ }^{1}$ \\ ${ }^{1}$ State Key Lab of Modern Optical Instrumentation, Zhejiang University, Hangzhou 310027, China \\ ${ }^{2}$ Zhejiang University of Science and Technology, Hangzhou 310023, China \\ *Corresponding author: Limin TONGＥ-mail: phytong@zju.edu.cn
}

\begin{abstract}
As a low-dimensional optical fiber with diameter close to or below the wavelength of light, optical micro/nanofiber (MNF) offers a number of favorable properties for optical sensing, which have been exploited in a variety of sensing applications, including physical, chemical, and biological sensors. In this paper we review the principles and applications of silica, glass, and polymer optical micro/nanofibers for physical and chemical sensing.
\end{abstract}

Keywords: Optical microfiber, optical nanofiber, tapered fiber, physical sensor, chemical sensor, review

\section{Introduction}

Optical methods are some of the oldest and best established sensing techniques. The development and improvement of optical sensors are driven by the continuous demand for simple, rapid, sensitive, and in-situ monitoring techniques in a broad range of areas including medical, pharmaceutical, environmental, defense, bioprocessing, and food industries. By using optical fibers as transduction elements, fiber-optic physical, chemical, and biosensors have been very successful in the past decades. With the rapid development of micro/nanotechnology, as well as the increasing demands on miniaturized sensors with higher performances, fiber optical sensors with small size, high sensitivity, fast response, high selectivity, and low detection limits are the current trends of sensing systems $[1,2]$.

In a standard optical fiber with diameter much larger than the wavelength of light, the intensity of evanescent field decays to almost zero at the outer surface of cladding. Thus, light propagating in these fibers is insensitive to the surroundings until it reaches the small-area endface, which limits the interaction between the light and the specimen and consequently the response of fiber optical sensor regarding sensitivity and sometimes the response time. With the advent of low-loss subwavelength-diameter micro/nanofibers (MNFs) [3], light can be guided along these tiny fibers with more flexibilities for optical sensing within the visible and near infrared spectral ranges. Due to their extremely small diameters, this kind of MNFs are capable of offering large fractions of evanescent waves and high-intensity surface fields [4, 5], making it highly sensitive to the index change of the surrounding medium by significantly enhancing the interaction between guided light and surrounding specimens $[5,6]$. In addition, these MNFs show excellent diameter uniformity and atomic-level sidewall smoothness, making them possible to guide light with low optical losses and high mechanical strength $[3,7,8]$. Therefore, these MNFs are promising for developing miniaturized fiber-optic sensors with high sensitivity and fast response.

Received: 24 October 2010 / Revised version: 22 November 2010

(C) The Authors(s) 2010.This article is published with open access at Springerlink.com 
So far a number of methods have been developed for fabricating MNFs [9-16], usually starting from materials such as glass and polymer. For example, Harfenist et al. [9] reported direct drawing of MNFs from solvated polymers. Sumetsky et al. [10] fabricated MNFs by drawing standard optical fiber in a microfurnace comprising a sapphire tube heated with a $\mathrm{CO}_{2}$ laser, Tong et al. [15] developed a method for directly drawing glass nanowires from bulk glasses by a sapphire fiber. More recently, Zhang et al. [16] reported a hydrofluoric acid flow etching method for the fabrication of low-loss, subwavelength-diameter biconical fiber tapers. The losses of as-fabricated tapers are less than $0.1 \mathrm{~dB} / \mathrm{mm}$, corresponding to an order of magnitude increase in the optical transmission over previous acid-etch techniques. Pricking et al. [17] reported a model that could accurately simulate the fabrication process of complex-shaped tapered fibers. Meanwhile, the waveguiding properties of MNFs including optical confinement [5], evanescent fields [5], waveguide dispersion [5, 18], roughness-induced radiation losses [19, 20], bending losses [21], and endface reflectivities [22] have been well studied by theoretical calculations and/or experimental investigation. Various MNF-based photonic devices [23-32] have been reported, among which MNF resonators [23-27] and interferometer [33] are particularly useful for sensing applications. For robust and stable operation, the MNF assembly can be embedded in low refractive index substrates [34, 35]. In addition, MNFs have been used for coupling light in and out of other sensing structures such as liquid-core optical ring-resonators [36], microdisks [37], and microspheres [38, 39].

Typical structures of optical MNF sensors introduced in this paper are illustrated in Fig. 1. Figure 1(a) depicts the simplest uncoated MNF sensor, in which the thickness of MNF is usually close to or less than the wavelength of light. Since high fractional guiding mode of MNF propagates outside the fiber, transmission intensity of MNF depends on the properties of ambient medium, which has been applied for refractive index (RI), humidity, and chemical/ biological sensing. Sometimes, the surface of MNF can be coated with reagents wider sensing applications. Figure 1(b) illustrates a tapered fiber tip sensor, which is usually coated with a sensitive film to identify chemical or biological species. It has been used for RI sensing and some fluorescence based chemical/biological sensing. Figure 1(c) illustrates evanescent coupled nanowire-MNF sensor, which is in principle similar to Fig. 1(a), but offers more flexibility for functionalizing the sensing elements, and has been applied to polymer or semiconductor nanowire optical sensors. While sensors in Figs. 1(a)-1(c) employ intensity-dependent scheme, those in Figs. 1(d)-1(f) depend on optical phase or path. Figure 1(d) shows a sensing structure relying on nanowire-MNF Mach-Zehnder interferometer (MZI). Figure 1(e) illustrates optical sensors based on MNF loop/knot/coil resonators that are assembled by micromanipulation. Figure 1(f) shows a liquid-core optical ring-resonator sensor using MNF for input and output connections.
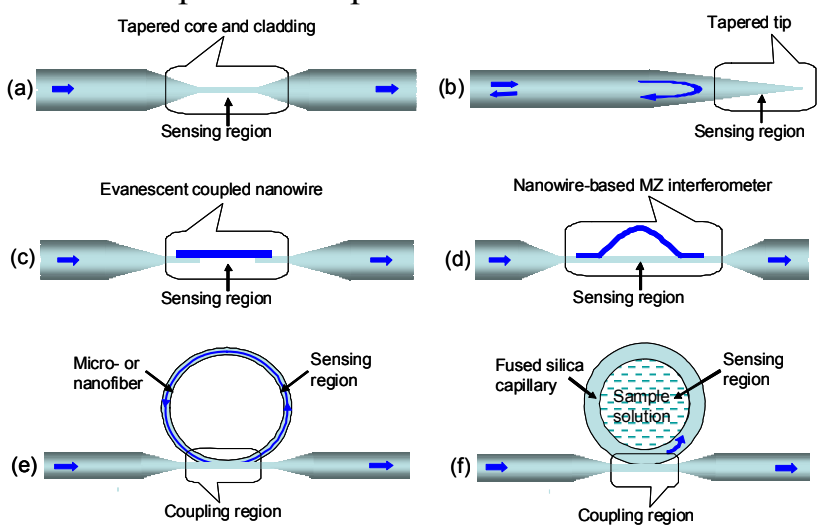

Fig. 1 Illustration of typical structures of optical MNF sensors: (a) straight MNF sensor, (b) tapered tip MNF sensor, (c) evanescent coupled nanowire MNF sensor, (d) nanowire-based MZI MNF sensor, (e) MNF loop/knot/coil resonator sensor, and (f) liquid-core optical ring-resonator sensor.

While considerable research efforts have been 
reported on physical, chemical, and biological sensors using one-dimensional optical structures ranging from optical fibers, nanowires to planar waveguides, this review focuses on glass and polymer optical MNF sensors. MNF lasers, optical nonlinear effects in MNFs, and other applications are not included. We classifies these sensors into the following categories: physical sensors, chemical sensors, and biological sensors.

\section{Physical sensors}

This section introduces MNF based physical sensors for RI, humidity, temperature, accelerator, and gyroscope.

\subsection{RI sensor}

RI is one of the basic physical properties in optical sensing. Most commonly it is used to measure the concentration of a solute in an aqueous solution. For example, RI can be used to determine the concentration of sugar or proteins. Thus, RI measurement is very important for chemical and biological sensing. A number of MNF-based RI sensors were reported recently. According to the sensor structure, we classifies these sensors into the following categories: straight MNF RI sensor, twist MNF RI sensor, looped or coiled MNF RI sensor, and MZI based MNF RI sensor.

\subsubsection{Straight MNF RI sensor}

As shown in Fig. 2 (a), Polynkin et al.[40] reported a simple optical sensing device capable of measuring the RI of liquids propagating in microchannels. The sensor was based on a MNF immersed in polydimethylsiloxane (PDMS). A channel for liquid analyte was fabricated in the immediate vicinity of taper waist. Evanescent fields propagating through the tapered section of the fiber extended into the channel, making it highly sensitive to the RI change of liquid. The sensor could operate both as a highly responsive on-off device and in the continuous measurement mode, with an estimated accuracy of $5 \times 10^{-4}$ for RI measurement.
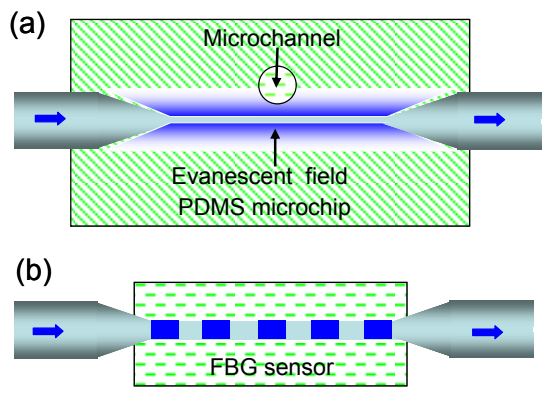

(c)

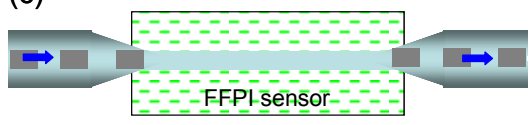

Fig. 2 Illustration of straight MNF RI sensor: (a) MNF sensor with liquid channel, (b) single fiber Bragg grating sensor, and (c) fiber Fabry-Pérot interferometer (FFPI) sensor.

The sensing sensitivity of MNF sensor can be significantly increased by employing interferometric structure. As shown in Figs. 2(b) and 2(c), Liang et al. [41] reported a combined fiber Bragg grating (FBG) technology with a wet chemical etch-erosion procedure and demonstrated two types of RI sensors using single-mode optical fibers. The first index sensor was an etch-eroded single MNF FBG with a radius of $3 \mu \mathrm{m}$, which was used to measure the indices of four different liquids. The second index sensor device was an etch-eroded MNF FFPI with a radius of about $1.5 \mu \mathrm{m}$ and was used to measure the RIs of isopropyl alcohol solutions. Due to its narrower spectral feature, FFPI sensor has a higher sensitivity than FBG sensor and can detect an index variation of $1.4 \times 10^{-5}$.

Fang et al. [42] fabricated FBG in MNF by the use of femtosecond laser pulse irradiation. Such a grating could be directly exposed to the surrounding medium without etching or thinning treatment of fiber, thus possessing high RI sensitivity while maintaining excellent reliability. Depending on the fiber diameter, MNF grating supported a number of propagation modes, and the higher-order mode offered higher RI sensitivity. The RI sensitivity also depended on fiber diameter, usually a smaller diameter yielded a higher sensitivity. The highest sensitivity obtained was $231.4 \mathrm{~nm}$ per RI unit (RIU) at RI of 1.44 when the fiber diameterwas about $2 \mu \mathrm{m}$. 
More recently, Tai et al. [43] presented a RI sensor based on the change of optical confinement in a subwavelength tip (Fig.1 (b)). The optical spot was substantially increased when the environmental RI increased from 1.3 to 1.4. By measuring the intensity of low angular spectral components, an intensity sensitivity up to $8000 \%$ per RIU was achieved. The application for measuring concentrations of acids and evaporation rates of aqueous mixtures was demonstrated.

\subsubsection{Twist MNF RI sensor}

Xing et al. [44] reported flexible polymer MNFs with diameters down to $60 \mathrm{~nm}$ and lengths up to $500 \mathrm{~mm}$, fabricated by one-step drawing process from molten poly(trimethylene terephthalate) (PTT). Based on these MNFs, Zhu et al. [45] developed a tunable RI sensor by twisting two flexible PTT nanowires shown in Fig. 3. The sensor consisted of two input branches, a twisted coupling region, and two output branches. The changes of optical power due to variations in the surrounding medium around the twisted coupling region were measured in the output branches. The highest sensitivity of the sensor was $26.96 \mathrm{~mW} / \mathrm{RIU}$ and the detection limit on RI change was $1.85 \times 10^{-7}$.

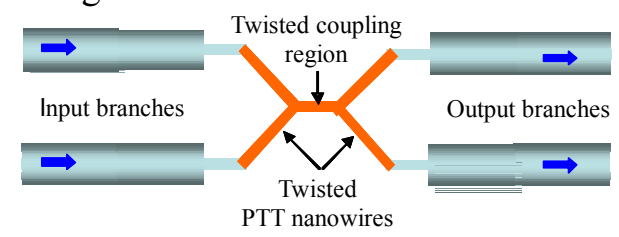

Fig. 3 Illustration of twisted MNF RI sensor.

\subsubsection{Looped or coiled MNF RI sensor}

High- $Q$ whispering gallery mode (WGM) microresonator such as MNF loop/knot/coil resonator shown in Fig. 4 (a) is highly sensitive to the change of the surrounding medium: when the RI changes, the resonant wavelength shifts (Fig. 4 (b)).

Shi et al. [46] set up a reliable theoretical model and optimized the structural parameters of microfiber loop resonators including the radius of microfiber, the radius of loop and the length of coupling region for higher sensitivity, wider dynamic measurement range, and lower detection limit. To show the sensing ability, they exploited the application of optical microfiber loop resonators in ambient RI sensing with a detection limit down to $10^{-5}$ RIU

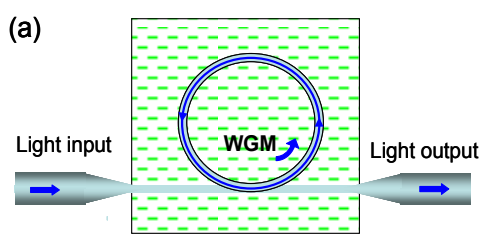

(b)

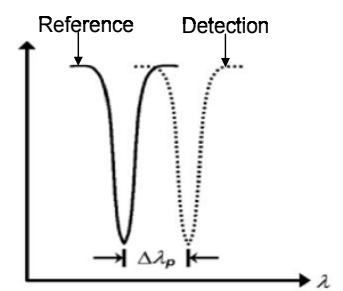

Fig. 4 (a) Schematic view of MNF loop/knot/coil resonator sensor and (b) schematic view of WGM resonant wavelength shift.

Guo et al. [32] demonstrated critical coupling condition in microfiber loops wrapped around a copper rod. The critical coupling condition was achieved by tuning the coupling coefficient to balance the circulation loss. Based on these copper-rod supported microfiber loops, Guo et al. [47] developed RI sensors. Due to the robustness of supported loop structure and the flexibility of obtaining critical coupling within a broad spectral range, these microfiber loops showed high sensitivity and high stability for sensing in both lowand high-concentration solutions with estimated sensitivity of RI measurement up to $1.8 \times 10^{-5}$ RIU.

$\mathrm{Xu}$ et al. [48] presented a robust and compact RI sensor based on a coated MNF microcoil resonator According to calculation, the sensitivity depended on fiber diameter and coating thickness. A sensitivity up to $700 \mathrm{~nm} / \mathrm{RIU}$ and a RI resolution down to $10^{-10}$ were predicted and consequently the RI sensor was experimentally demonstrated [49] with a sensitivity of about $40 \mathrm{~nm} / \mathrm{RIU}$. The authors also reported a RI sensor based on an embedded 
MNF loop resonator [50]. The sensitivity was studied in two typical configurations and its dependence on the fiber diameter and coating thickness was determined. More recently, Xu et al. [51] manufactured gratings in MNF and discussed their application to sensing. Gratings were manufactured by wrapping an MNF on a microstructured rod. This method avoids post-processing thin MNF and show flexibility: chirping can be realized by designing air hole size and position in the microstructured rod. By exploiting large evanescent field in an inner channel, microfluidic RI sensors with sensitivity of more than $10^{3} \mathrm{~nm} / \mathrm{RIU}$ can be achieved.

\subsubsection{MZI based MNF RI sensor}

Based on theoretical modeling, Wu et al. [52] proposed and investigated a highly-sensitive sensor employing a MNF-assembled MZI structure shown in Fig. 5. The sensor was used to measure the RIs of isopropyl alcohol solutions of different concentrations. A phase shift of guided mode, originating from the change of RI of ambient medium, was obtained. The MZI sensor based on MNFs exhibited the capability of measuring an index variation of $10^{-6}$ RIU.

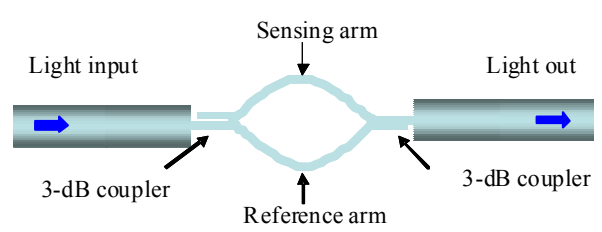

Fig. 5 Schematic of a MZI sensor assembled by two nanofibers.

\subsection{Temperature sensor}

As mentioned above, the narrow transmission resonance of a microring or microcoil resonator is very sensitive to the RI and length of the microring, which in turn is affected by temperature, pressure, and applied radiation. Therefore, the microring, microknot or microdisk resonator can be used for temperature sensing.

Sumetsky et al. [53] proposed an ultrafast direct contact temperature sensor using a microfiber loop resonator (MLR), with measured heating/cooling relaxation time of about $3 \mu \mathrm{s}$, which is in good agreement with theoretical calculation.

$\mathrm{Wu}$ et al. [54] reported two fiber-optic temperature sensors based on silica/polymer microfiber knot resonators (SMKR/PMKR). These sensors were composed of three layers: $\mathrm{MgF}_{2}$ crystal plate was adopted as the substrate and the sensing knots were covered by a thin $\mathrm{MgF}_{2}$ slab to keep it steady and immunity to environment fluctuations. Temperature sensitivity of SMKR was about $52 \mathrm{pm} /{ }^{\circ} \mathrm{C}$ within $30{ }^{\circ} \mathrm{C}$ around $700{ }^{\circ} \mathrm{C}$, while the sensitivity of PMKR is about $266 \mathrm{pm} /{ }^{\circ} \mathrm{C}$ within $20{ }^{\circ} \mathrm{C}$ around $80{ }^{\circ} \mathrm{C}$. Temporal response of SMKR and PMKR sensors were less than $1 \mathrm{~ms}$ and $5 \mathrm{~ms}$, respectively. These MNF knot resonators can be used as miniature high temperature sensors with fast response. Higher resolution can be anticipated with further improvement of $Q$ factor of the resonator.

Zeng et al. [55] experimentally proposed and demonstrated a compact temperature sensor based on MNF knot resonator that was placed on a plate glass substrate and coated with low-index polymer to keep the system robust. Sensitivities of this kind of temperature sensor of $0.27 \mathrm{~nm} /{ }^{\circ} \mathrm{C}$ in heating process (when temperature ranged from $28{ }^{\circ} \mathrm{C}$ to $140{ }^{\circ} \mathrm{C}$ ) and $-0.28 \mathrm{~nm} /{ }^{\circ} \mathrm{C}$ in cooling process (when temperature ranged from $135{ }^{\circ} \mathrm{C}$ to $25{ }^{\circ} \mathrm{C}$ ) were obtained. Temperature resolution of $0.5{ }^{\circ} \mathrm{C}$ was demonstrated and higher resolution was predicted with a high-resolution spectrometer.

Kou et al. [56] presented an ultra-small all-silica high temperature sensor based on a reflective Fabry-Perot modal interferometer (FPMI). The FPMI was made of a micro-cavity (about $4.4 \mu \mathrm{m}$ ) directly fabricated into a fiber taper probe less than $10 \mu \mathrm{m}$ in diameter. The sensing head was a miniaturized single mode-multimode fiber configuration without splicing. Sensing mechanism of FPMI is interference among reflected fundamental mode and excited high-order modes at the end-faces. Its temperature sensitivity is about $20 \mathrm{pm} /{ }^{\circ} \mathrm{C}$ near the wavelength of $1550 \mathrm{~nm}$. This 
kind of sensor can work in harsh environments with ultra-large temperature gradient.

Li et al. [57] demonstrated a high-sensitivity thermal sensor by coating a layer of PDMS on the surface of a silica toroidal microresonator on a silicon wafer. Possessing high- $Q$ WGMs, the PDMS-coated microresonator was highly sensitive to temperature change in the surroundings. To study the resonant wavelength shifts in PDMS-coated silica microtoroid, a MNF with diameter of about $1.5 \mu \mathrm{m}$ was used to couple the laser light into a PDMS-coated toroidal microresonator and also collected the light out. The measurement sensitivity of $0.151 \mathrm{~nm} / \mathrm{K}$ was one order of magnitude higher than pure silica microcavity sensors.

\subsection{Gyroscope and accelerometer}

Scheuer et al. [58] investigated the effect of rotation on propagation of electromagnetic waves in MNF microcoil resonator structures. It is shown that the combination of slow-light and conventional propagation mechanisms leads to an enhancement of orders of magnitudes of Sagnac phase shift and can be used for the realization of highly compact optical rotation sensors and gyroscopes.

Huo et al. [59] developed an optical accelerometer with a bulk-micromachined silicon proof mass and a MNF loop resonator (MLR) sensor. The MLR was fixed on the surface of cantilever beam. The proof mass and cantilever beam were fabricated with a two-mask process. An accelerometer with output sensitivity of $624.7 \mathrm{mV} / \mathrm{g}$ within a range of $+/-20 \mathrm{~g}$ was obtained.

\subsection{Current sensor}

Belal et al. [60] demonstrated a compact optical fiber microwire current sensor based on Faraday effect. In this current sensor, the change in the polarization azimuth of propagating optical field is proportional to the intensity of magnetic field induced by applied current. The compact sensor provides the ability to sense high frequency currents or magnetic fields in hazardous conditions. Moreover, it can monitor electrical breakdowns and coronas, where electric and magnetic field changes typically occur over rise time of about $1 \mathrm{~ns}$.

\subsection{Humidity sensor}

$\mathrm{Gu}$ et al. [61] reported a highly versatile nanosensors using polymer nanofibers or nanowires. For relative humidity (RH) sensing, a polyacrylamide (PAM) nanowire drawn from a PAM aqueous solution was employed for RH sensing based on a RH-dependent evanescent power leakage. As schematically illustrated in Fig. 6(a), a fiber taper drawn from a single-mode fiber with distal end about $500 \mathrm{~nm}$ in diameter is placed in parallel and close contact with one end of a polymer nanowire. Due to the strong evanescent coupling between the nanowire and the fiber taper, light can be efficiently launched into and picked up from the nanowire within a few micrometers' overlap. The estimated response time (baseline to $90 \%$ signal saturation) of the sensor is about $24 \mathrm{~ms}$ when RH jumps from 10\% to $75 \%$ and $30 \mathrm{~ms}$ when $\mathrm{RH}$ falls from $88 \%$ to $75 \%$, which are 1 or 2 orders of magnitude faster than those of existing RH sensors. The remarkably fast response of the sensor can be attributed to small diameter and large surface-to-volume ratio of the nanowire that enable rapid diffusion or evaporation of water molecules, as well as fast signal retrieval using optical approach.
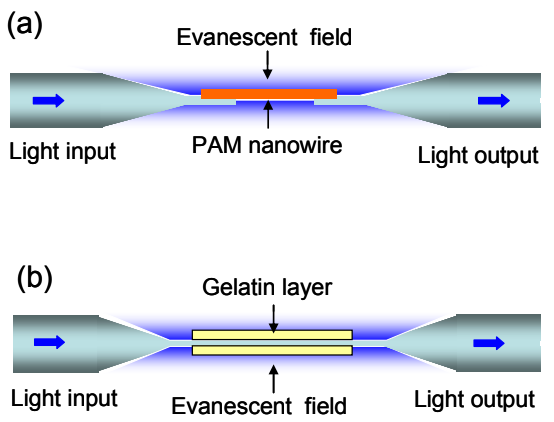

Fig. 6 (a) Schematic of evanescent coupled PAM nanowire humidity sensor and (b) schematic of a gelatin layer coated subwavelength-diameter tapered optical fiber sensor.

Zhang et al. [62] reported a RH sensor by using a subwavelength-diameter MNF coated with gelatin layer shown in Fig. 6(b). The sensing element was composed of a 680-nm-diameter MNF coated with a 
80-nm-thickness 8-mm-length gelatin layer, and was operated at a wavelength of $1550 \mathrm{~nm}$. When being exposed to moisture, the change in RI of the gelatin layer changed the mode field of the guided mode of the coated fiber, and converted a portion of power from guided mode to radiation mode, resulting in RH-dependent loss for optical sensing. The sensor was operated within a wide humidity range $(9 \% \mathrm{RH}$ - 94\% RH) with high sensitivity and good reversibility. Measured response time was about $70 \mathrm{~ms}$.

\section{Chemical sensors}

This section introduces some typical chemical sensors based on MNFs categorized by sensing mechanism and structure.

\subsection{Straight MNF gas sensor}

The sensing mechanism of chemical gas sensor is similar to humidity sensor. For example, hydrogen gas does not display intrinsic absorptions/emissions that could be used for optical sensing, so an additional functional coating should be used for hydrogen detection. Villatoro et al. reported hydrogen sensing $[63,64]$ using a palladium film coated MNF. When the sensor was exposed to hydrogen gas, the hydrogen interacted strongly with the platinum, resulting in change of optical transmission of fiber around $850 \mathrm{~nm}$ or $1550 \mathrm{~nm}$. By using thin MNF for enhancing evanescent-wave interaction between guided light and platinum coating, high sensitivity and fast response were obtained. For example, using a 1300-nm-diameter fiber with a 4-nm-thickness and 2-mm-length palladium film, a $10 \mathrm{~s}$ response time was observed [64], which was several times faster than that of conventional optical or electrical hydrogen sensors.

Compared with glass or oxide MNFs/nanowires, polymer nanowire, inherited from perm-selective nature and biocompatibility of polymer materials, offers a number of highly attractive advantages for sensing applications. The polymer single-nanowire optical sensor reported by $\mathrm{Gu}$ et al. [61] could also perform fast response gas sensing with high sensitivity. To tailor the nanowire for optical sensing, functional materials were doped into or blended with the solvated polymers before drawing process. The functionalized polymer nanowires were used for $\mathrm{NO}_{2}$ and $\mathrm{NH}_{3}$ detection down to sub-parts-per-million level with response time of 1 or 2 orders of magnitude faster than those of conventional sensors. Very recently, Gu et al. [65] demonstrated a single waveguiding polyaniline/polystyrene nanowire for highly selective detection of gas mixtures with a $\mathrm{NH}_{3}$ detection limit on parts-per-million level and relative humidity sensing ranging from $37 \%$ to $84 \%$.

\subsection{Surface absorption and fluorescence sensor}

The evanescent fields of MNF can be used to excite nearby molecules or particles that can then be detected via absorption. Warken et al. [66] reported ultra-sensitive surface absorption spectroscopy using sub-wavelength diameter MNFs. The absorption of particles on fiber surface was readily detected via the fiber transmission. The resulting absorption of a given surface coverage could be orders of magnitude higher than that in conventional surface spectroscopy. For example, a detection limit of sub-monolayer of 3,4,9,10-perylene-tetracarboxylic dianhydride (PTCDA) molecules was obtained, which revealed the agglomeration dynamics on a second to minutes timescale.

More recently, Stiebeiner et al. [67] presented spectroscopic measurements on PTCDA at ambient conditions by using guided mode of nanofiber for both excitation and fluorescence collection. The strong radial confinement and the pronounced evanescent field of guided light in optical nanofibers yielded favorable conditions for ultra-sensitive surface spectroscopy of molecules deposited on the fiber. It was found that surface coverage as small as 1 part per thousand of a compact monolayer still gave rise to fluorescence spectra with a good signal 
to noise ratio. Moreover, the authors analyzed and quantified the self-absorption effects due to reabsorption of emitted fluorescence light by circumjacent surface-adsorbed molecules distributed along the MNF.

\subsection{Evanescent spectrum based liquid sensor}

Generally, there are two types of evanescent-field-absorption sensing techniques, as shown in Fig. 7. The first approach measures the direct absorption of analytes in evanescent field of MNF, as shown in Fig. 7(a). The second approach measures indirect evanescent field absorption, in which a sensitive film is immobilized on fiber surface. When analytes interact with sensitive film, transmission changes will be observed, as shown in Fig. 7(b).

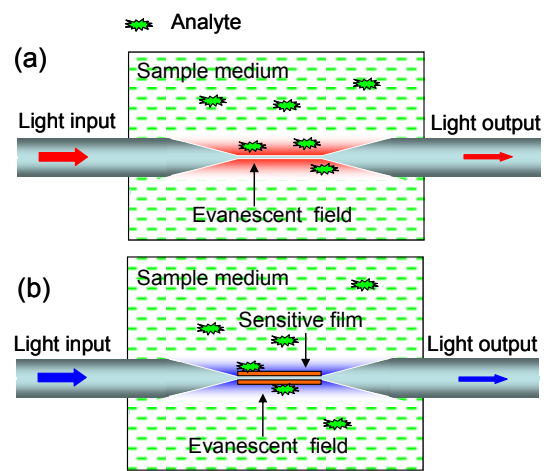

Fig. 7 Illustration of evanescent-field-absorption sensing approaches: (a) evanescent field absorption based on direct absorption of analytes in evanescent wave field of a heated stretching fiber taper and (b) indirect evanescent field absorption, a sensitive film is immobilized on the fiber taper surface, when analytes interact with sensitive film, transmission changes will be observed.

Based on the first technique, Vishnoi et al. [68] developed a spectrophotometer using tapered core multimode optical fiber, which was capable of identifying chemical species having the absorption peaks in visible region of the spectrum $(400 \mathrm{~nm}$ to $700 \mathrm{~nm}$ ). Aqueous dye solutions of different concentrations in pure and mixed form had been investigated. It was found that $1 \mu \mathrm{M}$ concentration of Rose Bengal could be detected, and a mixture of
Rose Bengal and Brilliant Green $\left(\lambda_{\max }: 625 \mathrm{~nm}\right)$ with $2 \mu \mathrm{M}$ concentration of each could easily be identified with their characteristic peaks.

For those analytes do not display intrinsic absorptions/emissions that could be used for optical sensing, they are usually detected indirectly. Based on the second technique, Baldini et al. [69] presented a $\mathrm{pH}$ sensor using a functionalized MNF. An acid-base indicator, thymol blue, was covalently immobilized on the fiber surface by means of a silylation process. The fiber surface was modified along a section of $8 \mathrm{~mm}$. Compared with a bare fiber, an enhancement in sensitivity of a factor 6 was observed in MNFs within the $\mathrm{pH}$ range from 1 to 2.5, and a sensitivity of $0.05 \mathrm{pH}$ units was attained.

Fluorescence measurements provide sensitive detection of chemicals. Since fluorescence intensity is proportional to excitation intensity, low concentration chemicals can be detected. Generally, there are two evanescent-field-fluorescence sensing techniques, as shown in Fig. 8.

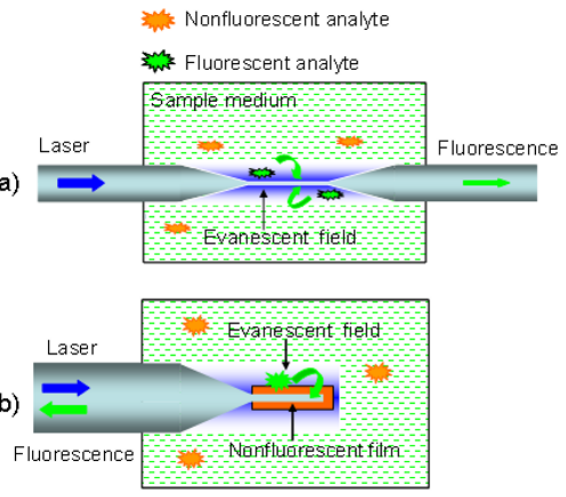

Fig. 8 Illustration of evanescent field fluorescence sensing approaches: (a) direct fluorescence method and (b) indirect method based on excited fluorescence intensity.

In the first approach, fluorescence is excited by evanescent field and then coupled back into MNF (Fig. 8(a)), known as direct method, in which evanescent field is used to deliver the excitation radiation as well as to collect the emission radiation. For example, when an excitation light of $460 \mathrm{~nm}$ is launched into a 3.69- $\mu \mathrm{m}$-diameter biconical taper (i.e., MNF) surrounded by a flourescein solution 
with concentration of $10 \mu \mathrm{M}$ to $60 \mu \mathrm{M}$, 516-nm-wavelength fluorescent light is collected by the same fiber, and the fluorescence signal is found proportional to the fluorescein concentration [70]. The results show the feasibility of measuring fluorescence through evanescent coupling, thus demonstrating the potential of using MNFs as fluorescence sensors for chemical and biological applications.

In the second approach, a nonfluorescent film is used to indirectly measure excited fluorescence intensity, as shown in Fig. 8(b). For example, in an ammonia microsensor reported recently [71], a fluorescent $\mathrm{pH}$ indicator was immobilized on the fiber tip. When ammonia interacted with the indicator, the fluorescence intensity increased. The ammonia concentration could be deduced by measuring the intensity of fluorescence. This microsensor showed a detection limit of $0.5 \mu \mathrm{g} / \mathrm{L}$. The dynamic range of the sensor matched perfectly with the concentration levels $(<25 \mu \mathrm{g} / \mathrm{L})$ that were known to be toxic for organisms. To reduce the response time of fluorescent fiber tip $\mathrm{pH}$ sensor, a highly hydrophilic nanostructure was fabricated on the tapered end using electrostatic layer-by-layer (LbL) assembly method, which accelerated water molecule and ion diffusion through the multilayer structure [72], resulting in five times faster in rise-time response from $\mathrm{pH} 3$ to $\mathrm{pH} 7$.

\subsection{Ring resonator based chemical vapor sensor}

Sun et al. [73] carried out simulations based on a four-layer Mie model to systematically analyze the sensing performance of ring resonator chemical vapor sensors. Two sensor configurations were investigated, in which a polymer layer was coated on either interior or exterior surface of a fused silica cylindrical ring resonator. Upon the interaction of polymer and vapor analyte, the RI and the thickness of polymer layer changed, leading to a spectral shift in the resonant modes that were supported by the ring resonator. The RI sensitivity and thickness sensitivity were studied as a function of polymer coating thickness and RI, the ring resonator size and wall thickness, and resonant mode order and polarization.

Besides the simulation results, the authors [74] have also experimentally developed rapid chemical-vapor sensors based on optofluidic ring resonators (OFRRs). The OFRR was a glass capillary whose circular wall supported the WGMs. The OFRR inner surface was coated with a vapor-sensitive polymer. A $1550 \mathrm{~nm}$ tunable diode laser scanned in wavelength was coupled into the WGM via an optical MNF in touch with the OFRR. An intensity dip at the fiber output end was used to indicate the WGM spectral position. When the vapor molecules passed through the OFRR and interacted with the polymer, the polymer RI changed, leading to a WGM spectral shift. The entire OFRR was placed on a plastic module covered by a glass slide to minimize temperature fluctuations. As a result, the OFRR exhibited subsecond detection and recovery time with a flow rate of only $1 \mathrm{~mL} / \mathrm{min}$, a few orders of magnitude lower than that of conventional optical vapor sensors. The detection limit was estimated to be $5.6 \times 10^{-6}$ RIU, more than ten times lower than other types of ring-resonator vapor sensors. Ethanol and hexane vapors were used as a model system, and chemical differentiation was demonstrated with different polymer coatings.

\section{Conclusions}

MNF can serve as a basic element for optical sensing or for light input/output in miniature photonic sensors. Due to their favorable properties of high fractional evanescent fields, low dimension, low loss, and high flexibility for optical sensing, MNF-based optical resonators may offer advantages of high sensitivity, fast response, small footprints, high spatial resolution, and low detection limits. In addition, the wavelength or nanoscale feature sizes of these fibers makes it possible to integrate the MNFs with microfluidic or nanofluidic chips for 
practical applications that require very small quantities (e.g., less than micro or nano liter level) of samples.

\section{Acknowledgment}

This work was supported by the National Natural Science Foundation of China under project No. 60907036 and No. 61036012, the Natural Science Foundation of Zhejiang Province, China under project No. Y1090021, the Fundamental Research Funds for the Central Universities under project No. 2010QNA5038 and the Specialized Research Fund for the Doctoral Program of Higher Education of China under project No. J20091636.

\section{Reference}

[1] A. Leung, P. M. Shankar, and R. Mutharasan, "A review of fiber-optic biosensors," Sensors and Actuators B: Chemical, vol. 125, no. 2, pp. 688-703, 2007.

[2] O. S. Wolfbeis, "Fiber-Optic Chemical Sensors and Biosensors," Analytical Chemistry, vol. 80, no. 12, pp. 4269-4283, 2008.

[3] J. Bures and R. Ghosh, "Power density of the evanescent field in the vicinity of a tapered fiber," Journal of the Optical Society America A, vol. 16, no. 8, pp. 1992-1996, 1999.

[4] L. M. Tong, J. Y. Lou, and E. Mazur, "Single-mode guiding properties of subwavelength-diameter silica and silicon wire waveguides," Optics Express, vol. 12, no. 6, pp. 1025-1035, 2004.

[5] J. Y. Lou, L. M. Tong, and Z. Z. Ye, "Modeling of silica nanowires for optical sensing," Optics Express, vol. 13, no. 6, pp. 2135-2140, 2005.

[6] L. M. Tong, R. R. Gattass, J. B. Ashcom et al., "Subwavelength-diameter silica wires for low-loss optical wave guiding," Nature, vol. 426, no. 6968, pp. 816-819, 2003.

[7] G. Brambilla, V. Finazzi, and D. J. Richardson, "Ultra-low-loss optical fiber nanotapers," Optics Express, vol. 12, no. 10, pp. 2258-2263, 2004.

[8] S. Leon-Saval, T. Birks, W. Wadsworth et al., "Supercontinuum generation in submicron fibre waveguides," Optics. Express, vol. 12, no. 13, pp. 2864-2869, 2004.

[9] S. A. Harfenist, S. D. Cambron, E. W. Nelson et al., "Direct drawing of suspended filamentary micro- and nanostructures from liquid polymers," Nano Letters, vol. 4, no. 10, pp. 1931-1937, 2004.

[10] M. Sumetsky, Y. Dulashko, and A. Hale, "Fabrication and study of bent and coiled free silica nanowires: Self-coupling microloop optical interferometer," Optics Express, vol. 12, no. 15, pp. 3521-3531, 2004.

[11] G. Brambilla, E. Koizumi, X. Feng et al., "Compound-glass optical nanowires," Electronics Letters, vol. 41, no. 7, pp. 400-402, 2005.

[12] L. M. Tong, J. Y. Lou, Z. Z. Ye et al., "Self-modulated taper drawing of silica nanowires," Nanotechnology, vol. 16, no. 9, pp. 1445-1448, 2005.

[13] G. Brambilla, F. Xu, and X. Feng, "Fabrication of optical fibre nanowires and their optical and mechanical characterisation," Electronics Letters, vol. 42, no. 9, pp. 517-519, 2006.

[14] L. Shi, X. F. Chen, H. J. Liu et al., "Fabrication of submicron-diameter silica fibers using electric strip heater," Optics Express, vol. 14, no. 12, pp. 5055-5060, 2006.

[15] L. M. Tong, L. L. Hu, J. J. Zhang et al., "Photonic nanowires directly drawn from bulk glasses," Optics Express, vol. 14, no. 1, pp. 82-87, 2006.

[16] E. J. Zhang, W. D. Sacher, and J. K. Poon, "Hydrofluoric acid flow etching of low-loss subwavelength-diameter biconical fiber tapers," Optics Express, vol. 18, no. 21, pp. 22593-22598, 2010.

[17] S. Pricking and H. Giessen, "Tapering fibers with complex shape," Optics Express, vol. 18, no. 4, pp. 3426-3437, 2010.

[18] J. Y. Lou, L. M. Tong, and Z. Z. Ye, "Dispersion shifts in optical nanowires with thin dielectric coatings," Optics Express, vol. 14, no. 16, pp. 6993-6998, 2006.

[19] G. Y. Zhai and L. M. Tong, "Roughness-induced radiation losses in optical micro or nanofibers," Optics Express, vol. 15, no. 21, pp. 13805-13816, 2007.

[20] A. V. Kovalenko, V. N. Kurashov, and A. V. Kisil, "Radiation losses in optical nanofibers with random rough surface," Optics Express, vol. 16, no. 8, pp. 5797-5806, 2008.

[21] H. K. Yu, S. S. Wang, J. Fu et al., "Modeling bending losses of optical nanofibers or nanowires," Applied Optics, vol. 48, no. 22, pp. 4365-4369, 2009.

[22] S. S. Wang, Z. F. Hu, H. K. Yu et al., "Endface reflectivities of optical nanowires," Optics Express, vol. 17, no. 13, pp. 10881-10886, 2009.

[23] M. Sumetsky, Y. Dulashko, J. M. Fini et al., "Optical microfiber loop resonator," Applied Physics Letters, vol. 86, no. 16, pp. 161108, 2005.

[24] L. M. Tong, J. Y. Lou, R. R. Gattass et al., "Assembly of silica nanowires on silica aerogels for microphotonic devices," Nano Letters, vol. 5, no. 2, pp. 259-262, 2005. 
[25] X. S. Jiang, L. M. Tong, G. Vienne et al., "Demonstration of optical microfiber knot resonators," Applied Physics Letters, vol. 88, no. 22, pp. 223501, 2006.

[26] X. D. Jiang, Y. Chen, G. Vienne et al., "All-fiber add-drop filters based on microfiber knot resonators," Optics Letters, vol. 32, no. 12, pp. 1710-1712, 2007.

[27] F. Xu and G. Brambilla, "Manufacture of 3-D microfiber coil resonators," IEEE Photonics Technology Letters, vol. 19, no. 17-20, pp. 1481-1483, 2007.

[28] Y. Chen, Z. Ma, Q. Yang et al., "Compact optical short-pass filters based on microfibers," Optics Letters, vol. 33, no. 21, pp. 2565-2567, 2008.

[29] G. Vienne, A. Coillet, P. Grelu et al., "Demonstration of a reef knot microfiber resonator," Optics Express, vol. 17, no. 8, pp. 6224-6229, 2009.

[30] S. S. Wang, Z. F. Hu, Y. H. Li et al., "All-fiber Fabry-Perot resonators based on microfiber Sagnac loop mirrors," Optics Letters, vol. 34, no. 3, pp. 253-255, 2009.

[31] P. Wang, L. Zhang, Z. Y. Yang et al., "Fusion Spliced Microfiber Closed-Loop Resonators," IEEE Photonics Technology Letters, vol. 22, no. 15, pp. 1075-1077, 2010.

[32] X. Guo, Y. H. Li, X. S. Jiang et al., "Demonstration of critical coupling in microfiber loops wrapped around a copper rod," Applied Physics Letters, vol. 91, no. 7, pp. 073512, 2007.

[33] Y. H. Li and L. M. Tong, "Mach-Zehnder interferometers assembled with optical microfibers or nanofibers," Optics Letters, vol. 33, no. 4, pp. 303-305, 2008.

[34] F. Xu and G. Brambilla, "Embedding optical microfiber coil resonators in Teflon," Optics Letters, vol. 32, no. 15, pp. 2164-2166, 2007.

[35] N. Lou, R. Jha, J. L. Domínguez-Juárez et al., "Embedded optical micro/nano-fibers for stable devices," Optics Letters, vol. 35, no. 4, pp. 571-573, 2010.

[36] I. M. White, H. Oveys, and X. Fan, "Liquid-core optical ring-resonator sensors," Optics Letters, vol. 31, no. 9, pp. 1319-1321, 2006.

[37] A. M. Armani and K. J. Vahala, "Heavy water detection using ultra-high- $Q$ microcavities," Optics Letters, vol. 31, no. 12, pp. 1896-1898, 2006.

[38] D. Keng, S. R. McAnanama, I. Teraoka et al., "Resonance fluctuations of a whispering gallery mode biosensor by particles undergoing Brownian motion," Applied Physics Letters, vol. 91, no. 10, pp. 103902, 2007.

[39] F. Vollmer, D. Braun, A. Libchaber et al., "Protein detection by optical shift of a resonant microcavity," Applied Physics Letters, vol. 80, no. 21, pp.
4057-4059, 2002.

[40] P. Polynkin, A. Polynkin, N. Peyghambarian et al., "Evanescent field-based optical fiber sensing device for measuring the refractive index of liquids in microfluidic channels," Optics Letters, vol. 30, no. 11, pp. 1273-1275, 2005.

[41] W. Liang, Y. Y. Huang, Y. Xu et al., "Highly sensitive fiber Bragg grating refractive index sensors," Applied Physics Letters, vol. 86, no. 15, pp. 151122, 2005.

[42] X. Fang, C. R. Liao, and D. N. Wang, "Femtosecond laser fabricated fiber Bragg grating in microfiber for refractive index sensing," Optics Letters, vol. 35, no. 7, pp. 1007-1009, 2010.

[43] Y. H. Tai and P. K. Wei, "Sensitive liquid refractive index sensors using tapered optical fiber tips," Optics Letters, vol. 35, no. 7, pp. 944-946, 2010.

[44] X. Xing, Y. Wang, and B. Li, "Nanofibers drawing and nanodevices assembly in poly(trimethylene terephthalate)," Optics Express, vol. 16, no. 14, pp. 10815-10822, 2008.

[45] H. Zhu, Y. Wang, and B. Li, "Tunable Refractive Index Sensor with Ultracompact Structure Twisted by Poly(trimethylene terephthalate) Nanowires," ACS Nano, vol. 3, no. 10, pp. 3110-3114, 2009.

[46] L. Shi, Y. H. Xu, W. Tan et al., "Simulation of optical microfiber loop resonators for ambient refractive index sensing," Sensors, vol. 7, no. 5, pp. 689-696, 2007.

[47] X. Guo and L. M. Tong, "Supported microfiber loops for optical sensing," Optics Express, vol. 16, no. 19, pp. 14429-14434, 2008.

[48] F. Xu, P. Horak, and G. Brambilla, "Optical microfiber coil resonator refractometric sensor," Optics Express, vol. 15, no. 12, pp. 7888-7893, 2007.

[49] F. Xu and B. Gilberto, "Demonstration of a refractometric sensor based on optical microfiber coil resonator," Applied Physics Letters, vol. 92, no. 10, pp. 101126, 2008.

[50] F. Xu, V. Pruneri, V. Finazzi et al., “An embedded optical nanowire loop resonator refractometric sensor," Optics Express, vol. 16, no. 2, pp. 1062-1067, 2008.

[51] F. Xu, G. Brambilla, and Y. Q. Lu, “A microfluidic refractometric sensor based on gratings in optical fibre microwires," Optics Express, vol. 17, no. 23, pp. 20866-20871, 2009.

[52] P. H. Wu, C. H. Sui, and B. Q. Ye, "Modelling nanofiber Mach-Zehnder interferometers for refractive index sensors," Journal of Modern Optics, vol. 56, no. 21, pp. 2335-2339, 2009.

[53] M. Sumetsky, Y. Dulashko, J. M. Fini et al., "The microfiber loop resonator: Theory, experiment, and application," Journal of Lightwave Technology, vol. 
24, no. 1, pp. 242-250, 2006.

[54] Y. Wu, Y. J. Rao, Y. H. Chen et al., "Miniature fiber-optic temperature sensors based on silica/polymer microfiber knot resonators," Optics Express, vol. 17, no. 20, pp. 18142-18147, 2009.

[55] X. Zeng, Y. Wu, C. L. Hou et al., "A temperature sensor based on optical microfiber knot resonator," Optics Communications, vol. 282, no. 18, pp. 3817-3819, 2009.

[56] J. L. Kou, J. Feng, L. Ye et al., "Miniaturized fiber taper reflective interferometer for high temperature measurement," Optics. Express, vol. 18, no. 13, pp. 14245-14250, 2010.

[57] B. B. Li, Q. Y. Wang, Y. F. Xiao et al., "On chip, high-sensitivity thermal sensor based on high- $Q$ polydimethylsiloxane-coated microresonator," Applied Physics Letters, vol. 96, no. 25, pp. 251109 , 2010.

[58] J. Scheuer, "Fiber microcoil optical gyroscope," Optics Letters, vol. 34, no. 11, pp. 1630-1632, 2009.

[59] C. L. Hou, Y. Wu, X. Zeng et al., "Novel high sensitivity accelerometer based on a microfiber loop resonator," Optical Engineering, vol. 49, no. 1, pp. 014402, 2010.

[60] M. Belal, Z. Song, Y. Jung et al., "Optical fiber microwire current sensor," Optics Letters, vol. 35, no. 18, pp. 3045-3047, 2010.

[61] F. Gu, L. Zhang, X. Yin et al., "Polymer single-nanowire optical sensors," Nano Letters, vol. 8, no. 9, pp. 2757-2761, 2008.

[62] L. Zhang, F. X. Gu, J. Y. Lou et al., "Fast detection of humidity with a subwavelength-diameter fiber taper coated with gelatin film," Optics Express, vol. 16, no. 17, pp. 13349-13353, 2008.

[63] J. Villatoro, D. Luna-Moreno, and D. Monzon-Hernandez, "Optical fiber hydrogen sensor for concentrations below the lower explosive limit," Sensors and Actuators B-Chemical, vol. 110, no. 1, pp. 23-27, 2005.

[64] J. Villatoro and D. Monzon-Hernandez, "Fast detection of hydrogen with nano fiber tapers coated with ultra thin palladium layers," Optics Express, vol. 13, no. 13, pp. 5087-5092, 2005.

[65] F. X. Gu, X. F. Yin, H. K. Yu et al.,
"Polyaniline/polystyrene single-nanowire devices for highly selective optical detection of gas mixtures," Optics Express, vol. 17, no. 13, pp. 11230-11235, 2009.

[66] F. Warken, E. Vetsch, D. Meschede et al., "Ultra-sensitive surface absorption spectroscopy using sub-wavelength diameter optical fibers," Optics Express, vol. 15, no. 19, pp. 11952-11958, 2007.

[67] A. Stiebeiner, O. Rehband, R. Garcia-Fernandez et al., "Ultra-sensitive fluorescence spectroscopy of isolated surface-adsorbed molecules using an optical nanofiber," Optics Express, vol. 17, no. 24, pp. 21704-21711, 2009.

[68] G. Vishnoi, T. C. Goel, and P. K. C. Pillai, "Spectrophotometric studies of chemical species using tapered core multimode optical fiber," Sensors and Actuators B-Chemical, vol. 45, no. 1, pp. 43-48, 1997.

[69] F. Baldini, L. Ciaccheri, A. Falai et al., "Thymol blue immobilized on tapered fibres as an optical transducer for $\mathrm{pH}$ sensing," Chemical, Biochemical, and Environmental Fiber Sensors X, vol. 3540, pp. 28-33, 1999.

[70] P. J. Wiejata, P. M. Shankar, and R. Mutharasan, "Fluorescent sensing using biconical tapers," Sensors and Actuators B-Chemical, vol. 96, no. 1-2, pp. 315-320, 2003.

[71] K. Waich, T. Mayr, and I. Klimant, "Microsensors for detection of ammonia at ppb-concentration levels," Measurement Science \& Technology, vol. 18, no. 10, pp. 3195-3201, 2007.

[72] C. R. Zamarreno, J. Bravo, J. Goicoechea et al., "Response time enhancement of $\mathrm{pH}$ sensing films by means of hydrophilic nanostructured coatings," Sensors and Actuators B-Chemical, vol. 128, no. 1, pp. 138-144, 2007.

[73] Y. Sun and X. Fan, "Analysis of ring resonators for chemical vapor sensor development," Optics Express, vol. 16, no. 14, pp. 10254-10268, 2008.

[74] Y. Sun, S. I. Shopova, G. Frye-Mason et al., "Rapid chemical-vapor sensing using optofluidic ring resonators," Optics Letters, vol. 33, no. 8, pp. 788-790, 2008. 